\title{
Literature Review of the Usefulness of Smartphone for the Evaluation and Treatment of Mental Disorders
}

\author{
Seung-Ho Jang, MD¹, Young Sup Woo, MD, $\mathrm{PhD}^{2}$, Sang-Yeol Lee, MD, PhD', \\ Won-Myong Bahk, MD, PhD² \\ ${ }^{1}$ Department of Psychiatry, Wonkwang University School of Medicine, Iksan, ${ }^{2}$ Department of Psychiatry, College of Medicine, \\ The Catholic University of Korea, Seoul, Korea
}

\begin{abstract}
The drastic increase in mental illnesses is a global problem. Prompt improvement of symptoms through early intervention, analysis of drug efficacy, and evaluation of treatment compliance are key strategies to address this issue. However, the traditional diagnostic and therapeutic methods for mental illnesses entail assessments based on interviews or questionnaires, which require a lot of time and money. The widespread use of smartphones and their applications in recent years has made it easier to assess mental illnesses and access to treatment. However, the reliability and validity of use of smartphone applications as an assessment tool for mental illnesses has been questioned since these applications are largely based on the existing questionnaire assessment methods. To overcome these problems, use of biosensor-based smartphone applications for the diagnosis and assessment of mental illnesses is being studied. This study will introduce the current status of this approach and discuss the future prospects.
\end{abstract}

Keywords Smartphone; Psychiatry; Biosensing techniques; Immunoassay; Depression

\section{INTRODUCTION}

Over the past several decades, in the field of psychiatry, much effort has been devoted to diagnosing, evaluating, and treating mental illness more accurately and objectively. This has narrowed the gap with other medical fields and made diagnosis more accurate through the diagnostic classification system, and led to other achievements such as personalized care and the formulation of objective clinical guidelines. Traditionally, interviews, assessment scales, and clinical psychological tests have been used in the evaluation of mental symptoms to identify various changes in emotion, cognition, thinking, and behavior. Of late, however, studies on biological evaluation methods capable of accurate and objective evaluation in a short time are actively being conducted.
The smartphone is a mobile phone with advanced hardware and software functions that can perform complex functions like a personal computer. In recent years, owing to the surge in the use of mobile devices such as smartphones, tablets, and smartwatches, the evaluation of mental health on a daily basis has become easy. Mobile apps allow immediate assessment and are cost and time-effective by allowing users to store and use their mental health information on their smartphones. In a previous study, $72 \%$ of patients visiting the mental health department were using smartphones, and $50 \%$ of them were interested in using apps to check their mental status on a daily basis [1].

To date, studies have evaluated the feasibility and reliability of 'digital behavioral biomarkers' in major mental illnesses, examining mental status by analyzing

Received February 18, 2020, Revised February 24, 2020, Accepted February 24, 2020

Correspondence: Won-Myong Bahk, MD, PhD

Department of Psychiatry, Yeouido St. Mary's Hospital, College of Medicine, The Catholic University of Korea, 10 63-ro, Yeongdeungpo-gu, Seoul 07345 , Korea

TEL +82-2-3779-1250 FAX +82-2-780-6577 E-mail wmbahk@catholic.ac.kr ORCID https://orcid.org/0000-0002-0156-2510

Copyright $@$ by Korean Society for Affective Disorders. All Rights reserved.

This is an Open Access article distributed under the terms of the Creative Commons Attribution Non-Commercial License (http://creativecommons.org/licenses/ by-nc/4.0/) which permits unrestricted non-commercial use, distribution, and reproduction in any medium, provided the original work is properly cited. 
smartphone usage patterns [2]. Smartphones or wearable devices have also been used to evaluate physical activities, sleep patterns, and circadian rhythm, the critical and intrinsic parameters of mental illnesses. These new and advanced technologies can be authentic and continuous markers of individual behavior or digital footprints, and this paradigm shift can be a realistic bridge that narrows the gap between psychopathological behavior and psychopathological phenomena associated with mental illness. This is expected to help overcome various problems of traditional mental symptom evaluation methods.

Therefore, in this paper, we will discuss how smartphones and wearable devices have been used in mental health so far, and the methods associated with smartphone-based biosensing technology.

\section{USE OF SMARTPHONES IN PSYCHIATRY}

\section{Use in evaluation}

Despite clinical development, mental disorders are becoming highly chronic, leading to disability and contributing to the global burden of disease. In this context, smartphone technologies are presented as a novel and cost-effective intervention that helps in diagnosis, monitoring, and treatment of mental symptoms. Smartphones were mostly owned by younger people in the early stages of mental illness, and about $69 \%$ of first-episode psychosis patients had internet-enabled mobile devices [3]. Therefore, smartphones are being used in various ways to increase access to mental services such as screening high-risk groups, tracking outpatient symptoms, preventing recurrence, and improving drug compliance.

Previous studies have examined the prevalence and characteristics of various mental symptoms by applying existing survey tools to smartphone apps. BinDhim et al. [4] reported a prevalence of $82.5 \%$ and $66.8 \%$ for a cutoff of 11 and 15, respectively, on the Patient Health Questionnaire administered via smartphone apps to 8,241 respondents. In a study that administered the Korean version of the Mood Disorder Questionnaire via smartphone apps, $8.2 \%$ of the 27,159 participants were classified as high-risk for bipolar spectrum disorder, and there were differences according to previous psychiatric treatment or age group [5]. Jang et al.'s [6] survey using the Suicide Behaviors Questionnaire-Revised with the Center for Epidemiologic Studies-Depression scale via smartphone apps found that $25.7 \%$ of the respondents were at high risk of depression; the suicide risk factors included depression, being female, belonging to the 30 40 age group, and past psychiatric history. FaurholtJepsen et al. [7] collected self-monitored data (mood, sleep length, medication, activity, irritability, cognitive problems, alcohol consumption, stress, individualized early warning signs) of 61 patients with bipolar disorder using smartphone software (MONARCA I system) and compared the results to those of the 17-item Hamilton Depression Rating Scale and the Young Mania Rating Scale, which showed significant positive correlation. However, smartphone apps include potentially harmful content, such as describing access to lethal means or stimulating risky behavior, which makes cautious usage particularly important [8].

The reliability of the mental symptom questionnaires customized to smartphone apps has also been evaluated. Palmier-Claus et al. [9] evaluated the smartphone's capabilities as a platform for collecting clinical metrics in real time. Sixty-seven copies of the Positive and Negative Syndrome Scale (PANSS) and Calgary Depression Scale (CDS) were administered to 36 patients with schizophrenia for a week; there was a moderate to strong correlation between traditional paper and pencil rating, the PANSS, and the CDS. Chung et al. [10] developed the Korean version of the CESD-Revised and evaluated its usefulness among 20 participants. The evaluation of mental symptoms using smartphones is expected to increase rapidly owing not only to their convenience, promptness, and accessibility but also reliability.

\section{Use in treatment}

Therapeutic interventions using smartphone apps have been reported to be effective in improving mental symptoms by correcting lifestyles and reducing selfawareness and memory bias. In the FOCUS trial, in which 33 patients with schizophrenia/schizoaffective disorder were enrolled in self-management intervention and automatic surveys thrice a day for a month, medication compliance, social functioning, mood problems, auditory hallucinations, and sleep difficulties significantly improved [11]. Naslund et al. [12] evaluated the feasibility and acceptability of a weight loss program via a fitness tracker (Fitbit) among 10 patients with serious mental illnesses, and found a high daily usage of $89 \%$. A randomized controlled trial (RCT) among 118 
patients with depression divided the respondents into the test group using mobile self-monitoring apps to measure mood, stress, and daily activity $(\mathrm{n}=68)$ and the control group relying only on daily functions $(\mathrm{n}=46)$, and found that the group using mobile self-monitoring apps saw higher emotional self-awareness, decreased depression, and rapid improvement of mental symptoms [13]. In a study comparing the effects of a self-help [commitment therapy, mindfulness-based cognitive behavioral therapy (CBT)] app for suicidal ideation in 150 participants, not only the frequency and intensity of suicidal ideation but depression, anxiety, and impulsivity also decreased [14]. Larsen et al. [8] analyzed 49 mental health apps and found that the use of most resulted in increased support from family and friends, and that crisis support is the most effective suicide prevention strategy.

CBT was mainly used for therapeutic interventions using smartphones. In Watts et al.'s [15] RCT with 35 patients with depression, 15 patients were placed in the mobile CBT group and 20 in the computer CBT group. The RCT, which used a mobile app called 'Get Happy,' consisted of six sessions over eight weeks. As a result, depressive symptoms in both groups decreased after three months. A preliminary RCT using mobile CBT was also performed. For the trial, 23 participants were placed in either the mobile CBT group $(n=11)$ or the healthy control group $(n=12)$. The mobile CBT group attended three group meetings moderated by the psychologist and participated in self-monitoring, which clarified personal values and facilitated goal setting, relaxation, mindfulness, and acceptance. The results showed decreased depressive symptoms and improved overall health and working ability in the mobile CBT group compared to the control group [16]. In a study comparing the usage of a mobile CBT and mobile interpersonal therapy app in patients with social anxiety disorder, 52 patients were divided into the mobile CBT group $(n=27)$ and the mobile interpersonal therapy group $(n=25)$ and treatment effect was evaluated using the Liebowitz Social Anxiety Scale. The results showed that mobile CBT was more effective than mobile interpersonal therapy after three months [17]. Until now, the development of programs has focused on CBT, which can be conducted without face-to-face interaction between patient and therapist. However, such interactive treatment programs, which are in the pipeline, will dramatically change the way mental illness is treated.

\section{Smartphone as a mobile sensor}

Objective evaluations of illness activity can be automatically quantified using a smartphone and collected long-term without intrusiveness. Patients carry around a smartphone for most of the day, engaging in conversant or traceable activities. The data acquired by the smartphone, therefore, mainly are mainly related to patients' habitual behaviors. Illness activity is considered a sensitive and valid measure of prodromal symptoms in depression while conversely, increasing conversation is an important factor in anticipating the switch to hypomania [18]. Changes in social activity and physical activity/ psychomotor retardation are very important factors in the disease trajectory of patients with mental disorders [19]. In recent years, built-in smartphone sensors have been used to assess physical activity, location, movement, and audio environment, and the potential use as a mobile sensor is increasing through proximity with other objects and collaboration with other devices. LiKamWa et al. [20] reported the use of the 'MoodScope' in 32 participants. The MoodScope evaluates daily average mood through mobile sensing of short message service, email, phone call, application usage, web browsing, and location, and the results showed that the communication history and application usage had an accuracy of $94 \%$ in assessing users' average daily mood. Servia et al. evaluated self-reported moods and passively measured physical activity, sociability, and mobility among 18,000 participants via an Android app; mobile sensing of mood status was approximately $70 \%$ accurate. Burns et al. [21] compared 38 concurrent phone sensor values (e.g., global positioning system, ambient light, recent calls) and mood, emotions, cognitive/motivational states and concluded that 'Mobylyze!' is a scalable, feasible intervention.

There have also been studies that evaluated mental symptoms by analyzing vocal characteristics using a smartphone. In a study by Grunerbl et al. [22], state changes in 10 patients with bipolar disorder using smartphone sensing were compared with those of patients with bipolar disorder using four different sensing modalities (phone, sound, acceleration, location); recognition accuracies were $76 \%$, and state change detection precision and recall were $97 \%$. Faurholt-Jepsen et al. [23] measured voice feature, objective smartphone data on behavioral activities, and electronic monitored data among 28 patients with bipolar disorder using smartphones and 
found that voice features were more accurate, sensitive, and specific in manic or mixed states area under the curve $(\mathrm{AUC}=0.89)$ than in depressive states $(\mathrm{AUC}=0.78)$.

Studies have also evaluated eye movement and blinking using wearable electrooculography glasses. Laksana et al. [24] used facial expression (intensity and frequency of smiling, frowning behavior, eyebrow raises, head movement, etc.) to detect suicidal ideation. Valstar et al. [25] analyzed facial features of audio cues to assess the symptom severity of patients with depression. Such smartphone mobile sensing technologies are expected to be applied not only to learning about user behavior patterns but also to potential predictions about various mental symptoms such as patients' mood and well-being.

\section{Smartphone as a biosensor platform}

\section{1) Heart rate variability}

Heart rate variability (HRV) is defined as the variation between the input from the sympathetic and parasympathetic division of the autonomic nervous system (ANS) and heartbeat over time [26]. HRV is known as a potential diagnostic and prognostic biomarker of depression, and it is possible to measure acute HRV with only 120s of electrocardiographic recording [27]. Usually, high frequency HRV reflects parasympathetic activity and low frequency HRV reflects a combination of parasympathetic and sympathetic activity. Previous studies have consistently reported a decrease in HRV in depression [28], bipolar disorder, and schizophrenia [29]. Lin et al. [30] reported that symptoms of depression and anxiety, sleep quality, and pre-sleep arousal were significanctly improved by HRV-biofeedback among patients with major depressive symptoms. Dysregulation of autonomic homeostasis has been reported in schizophrenia, and this is associated with the hypoactivity or hyperactivity of the ANS [31]. Smartphones measure HRV by using the light-emitting diode (LED) of the inbuilt flash as the light source and the complementary metal oxide semiconductor (CMOS) camera as the light sensor to perform photoplethysmography, and is also used as a pulse wave recorder. When Peng et al. [32] evaluated frequency-domain and nonlinear parameters, the parameters of HRV, among 30 normal participants using photoplethysmographs of smartphones, the results showed a significantly high correlation with laboratory ECG results. Matsumura et al. [33] measured beat-by-beat HR and normal pulse volume via smartphone using the
iPhysioMeter app among 12 participants, and compared the results to that of conventional laboratory measures; the comparison showed the iPhysioMeter app to be very effective in evaluating absolute levels of heart rate and relative changes in normal pulse volume. Unfortunately, few studies have evaluated mental symptoms by analyzing HRV through smartphones. However, smartphones are expected to be used for evaluation and treatment of various mental symptoms owing to their ease of use, prompt confirmation of results, and objectivity of evaluation.

\section{2) Electroencephalogram}

Electroencephalogram (EEG) complexity is considered an indicator that discriminates the variability of the electrophysiological pattern by functional brain activities. To date, EEG characteristics have been studied in major affective disorders, schizophrenia, alcoholism, and bipolar mood disorder [34,35], and in previous studies, differences in background EEG rhythm have been found in obsessive compulsive disorder while differences in alteration in the mean alpha rhythm and asymmetry were found in schizophrenia [36]. Reduction of rapid eye movement latency in sleep EEG has been suggested as a specific marker of depression among patients with mania, schizophrenia, schizoaffective disorder, panic disorder, eating disorders, obsessive compulsive disorder, and sexual impotence [37]. McKenzie et al. [38] compared the results derived from the smartphone brain scanner-2 (SBS2), a smartphone based-EEG, with standard clinical EEG results among 205 participants and found that the SBS2 shows high specificity and sensitivity in detecting epileptiform abnormality. Cao et al. [39] used ketamine for 55 treatment-resistant depression patients and evaluated its effects using wearable EEG (Mindo-4S Jellyfish); they found increased alpha power and decreased alpha asymmetry in the responder. Lin et al. [40] conducted a 90-minute sustained-attention driving task with 15 participants using the Mindo system and wearable EEG, and found that EEG activities were highly correlated with variations in vigilance.

\section{3) Electrodermal activity}

Electrodermal activity (EDA) refers to changes in skin electrical properties, and reflects cognitive and emotional processing in the central nervous system. Heightened EDA represents negative symptoms and poor functional outcomes in patients with schizophrenia [41], and it has 
been proposed as a tool to distinguish between bipolar disorder and mood states (depression, hypomania, euthymia) [42]. In addition, as EDA is a risk marker of suicide, Jandl et al. [43] reported a significantly lower EDA habituation rate in suicide attempt patients, and Thorell et al. [44] reported that EDA signals reflect the suicide propensity of depression patients.

\section{4) Immunoassays}

Cortisol is the best-known biomarker to assess psychological stress. While interview evaluation is generally the method for quick and accurate diagnosis of stress, EEG, electrocardiograms, and body temperature are also used. Although they have the advantage of showing seasonal sensitivity and resolution of physiological response to external stimuli, they are often not suitable for public or point-of-care testing as they are relatively complicated and require bulky equipment. There is extensive ongoing research on lateral flow assay (LFA) as a way to overcome these problems [45]. LFA can be quantitatively analyzed without special tools, but it is difficult to quantify. To date, there have been proposals for methods of measuring cortisol using smartphones, most of which involve using sensitive colorimetric LFA strips to quantify cortisol in saliva. Simple LEDs have been used to shine light on the test strips, quantifying their intensity, with the CMOS image sensor analyzing mental status using a digital signal embedded in the smartphone [46]. LFA strip images are searched in real time and the results are interpreted by converting the red, green, and blue signal data into hue and brightness values [47]. The curvefitting method is mainly used for quantification. Choi et al. [48] developed a smartphone-based measurement system consisting of a smartphone, holder, and lateral flow immune strip, and used a smartphone camera and a light source to read colorimetric signals from the LFA. The results showed a high correlation between the color intensity of the test line and the cortisol concentration in the range of 1-100 $\mathrm{ng} / \mathrm{mL}$. Ray et al. [49] combined LFA with a portable imaging device and transmitted a salivary cortisol result to a smartphone within 15 minutes. Guler et al. [50] analyzed the LFA-based noncompetitive cocaine assay using a mobile app and detected it in the $0.01-1.0 \mu \mathrm{g} / \mathrm{mL}$ range from cocaine added synthetic saliva samples. A high level of accessibility is required for rapid and accurate assessment of mental symptoms, and as smartphone-based immunoassay technology can be used as a point-of-care tool, we anticipate its extensive usage in future assessments of mental symptoms, such as screening of high-risk groups and evaluation of drug efficacy.

\section{DISCUSSION}

Despite advances in psychiatry, the development of measures for the objective and rapid evaluation of mental symptoms is still insufficient. Given the nature of mental illness, there has been the constant question of how to evaluate and manage patients who avoid socializing and have trouble in interpersonal relationships. In addition, it is critical to increase access to medical care as mental illness is prevalent among people with low socioeconomic status and marginalized groups (e.g., military, elderly, children, immigrants, etc.). Therefore, smartphone or wearable device technology that can evaluate mental symptoms easily is very important. Innovation in digital healthcare has already begun in many directions. The development of digital technology will bring about major changes in the future of psychiatry, and such changes are already taking place in various fields. As described earlier, the technology for collecting and analyzing behavioral, psychological, and social markers presents new possibilities for mental healthcare. Smartphone, wearable device, genetic information analysis technology, and so on enables healthcare big data to be measured without limitation of time or place, and the development of healthcare platforms and the cloud enables real-time storage and management of these data. Such data will be analyzed within existing healthcare systems or through new technologies such as artificial intelligence, and will provide new insights for healthcare and disease treatment. However, existing studies in the field of mental healthcare have not progressed beyond analyzing biological markers in the laboratory. Future issues, therefore, revolve around how to integrate these biomarkers into smartphones or wearable devices and how to increase confidence in the results measured in this way. In general, future technologies in the field of mental healthcare are expected to proceed in two directions. The first is obtaining personal daily information and biosignals through a sensor built in a smartphone or a wearable device; this requires the development of various biomarkers as well as sensors and apps that can detect them. The second direction is the development of technology that can connect additional devices to the 
smartphone to obtain more precise and versatile data. It is expected to be useful for objective and accurate evaluations of mental symptoms by analyzing not only the current EEG, HRV, and EDA but also saliva, blood, sweat, and various other human derivatives. This will enable patients' daily information and biomarkers to be acquired in real time, in turn enabling comprehensive evaluation of the psychological state of patients, which vary with time and place. Eventually, it may be possible to quell the doubts about the reliability and objectivity of traditional mental symptom evaluation methods based on self-report or the internet.

However, these techniques come with various limitations. First, data acquired through smartphones or wearable devices can be easily stored and converted, which may lead to problems related to personal information breach. A smartphone or wearable device poses the risk of privacy invasion as it records a lot of information about an individual's daily life, and if sensitive information related to an individual's mental health is used for foul purposes, it may have the adverse effect of strengthening the social stigma associated with mental illness. Second, indiscriminate research on various biomarkers that are still in the experimental stage and have not yet been proven can add confusion to accurately assessing mental symptoms. Third, most of the studies reported so far have had small sample sizes and been conducted in nonclinical environments, and even when evaluating the therapeutic effect, the time frame has been short. Therefore, there must be additional clinical studies on sensing technology, and there needs to be continuous technological development based on scientifically proven theories. Lastly, it is difficult to evaluate biomarkers over the long term owing to the temporality and variability of the use of smartphones or wearable devices. Future research should consider methods to keep users engaged while using smartphones over a long period of time.

\section{CONFLICTS OF INTEREST}

The authors have nothing to disclose.

\section{ORCID}

\author{
Seung-Ho Jang \\ https://orcid.org/0000-0002-3479-0552
}

\author{
Young Sup Woo \\ https://orcid.org/0000-0002-0961-838X \\ Sang-Yeol Lee \\ https://orcid.org/0000-0003-1828-9992 \\ Won-Myong Bahk \\ https://orcid.org/0000-0002-0156-2510
}

\section{REFERENCES}

1. Torous J, Friedman R, Keshavan M. Smartphone ownership and interest in mobile applications to monitor symptoms of mental health conditions. JMIR Mhealth Uhealth 2014;2:e2.

2. Hidalgo-Mazzei D, Young AH, Vieta E, Colom F. Behavioural biomarkers and mobile mental health: a new paradigm. Int J Bipolar Disord 2018;6:9.

3. Lal S, Dell'Elce J, Tucci N, Fuhrer R, Tamblyn R, Malla A. Preferences of young adults with first-episode psychosis for receiving specialized mental health services using technology: a survey study. JMIR Ment Health 2015;20:e18.

4. BinDhim NF, Shaman AM, Trevena L, Basyouni MH, Pont LG, Alhawassi TM. Depression screening via a smartphone app: cross-country user characteristics and feasibility. J Am Med Inform Assoc 2015;22:29-34.

5. Woo YS, Bahk WM, Hong J, Yoon BH, Hwang TY, Kim $\mathrm{MD}$, et al. Use of a smartphone application to screen for bipolar spectrum disorder in a community sample. Health Informatics J 2016;22:779-88.

6. Jang SH, Woo YS, Hong JW, Yoon BH, Hwang TY, Kim $\mathrm{MD}$, et al. Use of a smartphone application to screen for depression and suicide in South Korea. Gen Hosp Psychiatry 2017;46:62-7.

7. Faurholt-Jepsen M, Vinberg M, Frost M, Christensen EM, Bardram JE, Kessing LV. Smartphone data as an electronic biomarker of illness activity in bipolar disorder. Bipolar Disord 2015; 17:715-28.

8. Larsen ME, Nicholas J, Christensen H. A systematic assessment of smartphone tools for suicide prevention. PLoS One 2016;11:e0152285.

9. Palmier-Claus JE, Ainsworth J, Machin M, Barrowclough C, Dunn G, Barkus E, et al. The feasibility and validity of ambulatory self-report of psychotic symptoms using a smartphone software application. BMC Psychiatry 2012;17:172.

10. Chung K, Jeon MJ, Park J, Lee S, Kim CO, Park JY. Development and evaluation of a mobile-optimized daily selfrating depression screening app: a preliminary study. PLoS One 2018;13:e0199118.

11. Ben-Zeev D, Brenner CJ, Begale M, Duffecy J, Mohr DC, Mueser KT. Feasibility, acceptability, and preliminary efficacy of a smartphone intervention for schizophrenia. Schizophr Bull 2014;40:1244-53.

12. Naslund JA, Aschbrenner KA, Bartels SJ. Wearable devices 
and smartphones for activity tracking among people with serious mental illness. Ment Health Phys Act 2016;10:10-7.

13. Reid SC, Kauer SD, Hearps SJ, Crooke AH, Khor AS, Sanci LA, et al. A mobile phone application for the assessment and management of youth mental health problems in primary care: a randomised controlled trial. BMC Fam Pract 2011;12:131.

14. Shand FL, Ridani R, Tighe J, Christensen H. The effectiveness of a suicide prevention app for indigenous Australian youths: study protocol for a randomized controlled trial. Trials 2013;14:396.

15. Watts S, Mackenzie A, Thomas C, Griskaitis A, Mewton L, Williams A, et al. CBT for depression: a pilot RCT comparing mobile phone vs. computer. BMC Psychiatry 2013;13:49.

16. Lappalainen P, Kaipainen K, Lappalainen R, Hoffrén H, Myllymäki T, Kinnunen ML, et al. Feasibility of a personal health technology-based psychological intervention for men with stress and mood problems: randomized controlled pilot trial. JMIR Res Protoc 2013;2:e1.

17. Dagöö J, Asplund RP, Bsenko HA, Hjerling S, Holmberg A, Westh $\mathrm{S}$, et al. Cognitive behavior therapy versus interpersonal psychotherapy for social anxiety disorder delivered via smartphone and computer: a randomized controlled trial. J Anxiety Disord 2014;28:410-7.

18. Faurholt-Jepsen M, Vinberg M, Christensen EM, Frost M, Bardram J, Kessing LV. Daily electronic self-monitoring of subjective and objective symptoms in bipolar disorder--the MONARCA trial protocol (MONitoring, treAtment and pRediCtion of bipolAr disorder episodes): a randomised controlled single-blind trial. BMJ Open 2013;3:e003353.

19. Kupfer DJ, Weiss BL, Foster G, Detre TP, McPartland R. Psychomotor activity in affective states. Arch Gen Psychiatry 1974;30:765-8.

20. LiKamWa R, Liu Y, Lane ND, Zhong L. MoodScope: building a mood sensor from smartphone usage patterns. Paper presented at: Proceedings of the 11th Annual International Conference on Mobile Systems, Applications, and Services; 2013 Jun 25-28; Taipei, Taiwan. Taipei: 2013. p. 389-401.

21. Burns MN, Begale M, Duffecy J, Gergle D, Karr CJ, Giangrande E, et al. Harnessing context sensing to develop a mobile intervention for depression. J Med Internet Res 2011;13:e55.

22. Grünerbl A, Muaremi A, Osmani V, Bahle G, Ohler S, Tröster G, et al. Smartphone-based recognition of states and state changes in bipolar disorder patients. IEEE J Biomed Health Inform 2015;19:140-8.

23. Faurholt-Jepsen M, Busk J, Frost M, Vinberg M, Christensen EM, Winther O, et al. Voice analysis as an objective state marker in bipolar disorder. Transl Psychiatry 2016;6:e856.

24. Laksana E, Baltrušaitis T, Morency LP, Pestian J. Investigating facial behavior indicators of suicidal ideation. Paper presented at: 12th IEEE International Conference on Auto- matic Face \& Gesture Recognition (FG 2017); 2017 May 30- Jun 3; Washington, DC, USA. IEEE, 2017.

25. Valstar M, Schuller B, Smith K, Almaev T, Eyben F, Krajewski J, et al. AVEC 2014: 3D dimensional affect and depression recognition challenge. Paper presented at: Proceedings of the 4th International Workshop on Audio/Visual Emotion Challenge; 2014 Nov 3-7; Orlando, FL, USA. pp. 3-10.

26. Ernst G. Heart-rate variability-more than heart beats? Front Public Health 2017;5:240.

27. Munoz ML, van Roon A, Riese H, Thio C, Oostenbroek E, Westrik I, et al. Validity of (ultra-)short recordings for heart rate variability measurements. PLoS One 2015;10:e0138921.

28. Kemp AH, Quintana DS, Gray MA, Felmingham KL, Brown K, Gatt JM. Impact of depression and antidepressant treatment on heart rate variability: a review and metaanalysis. Biol Psychiatry 2010;67:1067-74.

29. Quintana DS, Westlye LT, Kaufmann T, Rustan ØG, Brandt $\mathrm{CL}$, Haatveit B, et al. Reduced heart rate variability in schizophrenia and bipolar disorder compared to healthy controls. Acta Psychiatr Scand 2016;133:44-52.

30. Lin IM, Fan SY, Yen CF, Yeh YC, Tang TC, Huang MF, et al. Heart rate variability biofeedback increased autonomic activation and improved symptoms of depression and insomnia among patients with major depression disorder. Clin Psychopharmacol Neurosci 2019;17:222-32.

31. Fujibayashi M, Matsumoto T, Kishida I, Kimura T, Ishii C, Ishii $\mathrm{N}$, et al. Autonomic nervous system activity and psychiatric severity in schizophrenia. Psychiatry Clin Neurosci 2009;63:538-45.

32. Peng RC, Zhou XL, Lin WH, Zhang YT. Extraction of heart rate variability from smartphone photoplethysmograms. Comput Math Methods Med 2015;2015:516826.

33. Matsumura K, Rolfe P, Yamakoshi T. iPhysioMeter: a smartphone photoplethysmograph for measuring various physiological indices. Methods Mol Biol 2015;1256:30526.

34. Prichep LS, John ER. QEEG profiles of psychiatric disorders. Brain Topogr 1992;4:249-57.

35. Merrin EL, Floyd TC. Negative symptoms and EEG alpha activity in schizophrenic patients. Schizophr Res 1992;8:1120.

36. Prichep LS, Mas F, Hollander E, Liebowitz M, John ER, Almas M, et al. Quantitative electroencephalographic subtyping of obsessive-compulsive disorder. Psychiatry Res 1993;50:25-32.

37. Kupfer DJ, Foster FG. Interval between onset of sleep and rapid-eye-movement sleep as an indicator of depression. Lancet 1972;2:684-6.

38. McKenzie ED, Lim AS, Leung EC, Cole AJ, Lam AD, Eloyan A, et al. Validation of a smartphone-based EEG among people with epilepsy: a prospective study. Sci Rep 2017;3:45567.

39. Zehong Cao, Chin-Teng Lin, Weiping Ding, Mu-Hong 
Chen, Cheng-Ta Li, Tung-Ping Su. Identifying ketamine responses in treatment-resistant depression using a wearable forehead EEG. IEEE Trans Biomed Eng 2019;66:1668-79.

40. Lin CT, Chuang CH, Huang CS, Tsai SF, Lu SW, Chen $\mathrm{YH}$, et al. Wireless and wearable EEG system for evaluating driver vigilance. IEEE Trans Biomed Circuits Syst 2014;8:165-76.

41. Schell AM, Dawson ME, Rissling A, Ventura J, Subotnik $\mathrm{KL}$, Gitlin MJ, et al. Electrodermal predictors of functional outcome and negative symptoms in schizophrenia. Psychophysiology 2005;42:483-92.

42. Greco A, Valenza G, Lanata A, Rota G, Scilingo EP. Electrodermal activity in bipolar patients during affective elicitation. IEEE J Biomed Health Inform 2014;18:1865-73.

43. Jandl M, Steyer J, Kaschka WP. Suicide risk markers in major depressive disorder: a study of electrodermal activity and event-related potentials. J Affect Disord 2010;123:13849.

44. Thorell LH, Wolfersdorf M, Straub R, Steyer J, Hodgkinson S, Kaschka WP, et al. Electrodermal hyporeactivity as a trait marker for suicidal propensity in uni- and bipolar depression. J Psychiatr Res 2013;47:1925-31.

45. Zainol Abidin AS, Rahim RA, Md Arshad MK, Fatin Nabi- lah MF, Voon CH, Tang TH, et al. Current and potential developments of cortisol aptasensing towards point-of-care diagnostics (POTC). Sensors (Basel) 2017; 17:1180.

46. Mudanyali O, Dimitrov S, Sikora U, Padmanabhan S, Navruz I, Ozcan A. Integrated rapid-diagnostic-test reader platform on a cellphone. Lab Chip 2012;12:2678-86.

47. Wu JH, Pen CC, Jiang JA. Applications of the integrated high-performance CMOS image sensor to range findersfrom optical triangulation to the automotive field. Sensors (Basel) 2008;8:1719-39.

48. Choi SY, Kim SC, Yang JS, Lee JH, Joo CM, Jung HI. Real-time measurement of human salivary cortisol for the assessment of psychological stress using a smartphone. Sens Bio-Sens Res 2014;2:8-11.

49. Ray E, Jain A, Abdullah S, Choudhury T, Erickson D. Personalized stress monitoring: a smartphone-enabled system for quantification of salivary cortisol. Pers Ubiquitous Comput 2018;22:867-77.

50. Guler E, Yilmaz Sengel T, Gumus ZP, Arslan M, Coskunol $\mathrm{H}$, Timur S, et al. Mobile phone sensing of cocaine in a lateral flow assay combined with a biomimetic material. Anal Chem 2017;89:9629-32. 